\title{
Alkoholkranke nicht im Stich lassen
}

\begin{abstract}
Mindestens jeder dritte stationäre Patient an einer Wiener Psychiatrie leidet an Alkoholkrankheit. An manchen internen Abteilungen und an Aufnahmestationen sowie Notambulanzen leidet fast die Hälfte der Patienten auch an schädlichem Alkoholgebrauch. Bei jedem vierten männlichen und jeder 15. weiblichen Patientin beim Allgemeinmediziner finden sich Alkoholfolgeschäden. Warum kommen nur $2 \%$ der Alkoholabhängigen in Österreich in fachgerechte Therapie? Ein Grund ist sicher die moralische Keule des „selber schuld“ und die auch von vielen Patienten geteilte Meinung des „man muss nur aufhören wollen“. Alkoholabhängigkeit wird sowohl in der Finanzierung der Behandlung, aber auch zum Beispiel im Unterbringungsgesetz oder im Österreichischen Strukturplan Gesundheit nicht als vollwertige Krankheit anerkannt. Gesellschaftliche Hintergründe erschweren eine sinnvolle und zielgerichtete medizinische Diagnose, Therapie und Prävention dieser Seuche unserer Zeit. Es ist ein Skandal, dass es in Wien keine ärztlich besetzten Ambulanzen für Alkoholkranke mehr gibt, sich diese Menschen also zu Recht im Stich gelassen fühlen.

Je früher der schädliche Alkoholgebrauch im Leben beginnt, desto wahrscheinlicher wird eine Alkoholkrankheit, die das Leben verkürzt und die Teilhabe am selbstbestimmten Leben verhindert. Bereits über $3 \%$ der 18-jährigen Österreicherln-
\end{abstract} nen leiden unter schädlichem Alkoholgebrauch, bei den 18-jährigen Österreicherlnnen, die auch rauchen, sind es $10 \%$ und bei den auch Cannabis konsumierenden Jugendlichen über $20 \%$. Der Tod der britischen Soulsängerin Amy Winehouse (nomen est omen) zeigte exemplarisch, wie viel

Der Tod der britischen Soulsängerin Amy Winehouse (nomen est omen) zeigt exemplarisch, wie viel Talent und Zukunft durch Alkohol ausgelöscht wird.

Talent und Zukunft durch Alkohol ausgelöscht

wird. Unser Autor vom Verein Dialog, Dr. Christian

Müller, beschreibt in diesem Zusammenhang die Reproduktion der Isolation durch Jugendliche mit Suchtmittelkonsum. Aber wie bringt man jungen Menschen das vernünftige Nicht-Gebrauchen von Alkohol und anderen Drogen bei? Wer verdient an der Ermöglichung und Förderung des Alkoholmissbrauchs junger Menschen, z. B. bei Maturareisen? Nach Angaben des Grazer Autors unseres Schwerpunkts Sucht, Prim Prof. Dr. Martin Kurz, wird immerhin die Hälfte des in Österreich konsumierten Alkohols von alkoholkranken Menschen konsumiert. Eine effektive Alkoholprävention würde also die Umsätze der alkoholassoziierten Wirtschaft halbieren!

Der nach wie vor moralisierende Umgang mit Alkoholabhängigen führt auch dazu, dass alkoholgeschädigte, insbesondere intellektuell beeinträchtigte Menschen nicht mehr rehabilitiert werden. Die innere Medizin behandelt Patienten mit COPD nicht erst nach dem Ende der Nikotinabhängigkeit. Die Endokrinologen behandeln den Diabetes mellitus nicht erst nach erfolgter Gewichtsreduktion. Warum wird bei aktuell alkoholabhängigen Menschen nicht versucht, wenigstens den Schaden durch den Alkoholkonsum zu minimieren, in dem z. B. regelmäßige Vitamin B1-Injektionen verabreicht werden. Die Pflegeheimeinweisung der vielen tausend alkoholbedingten Korsakowpatienten Österreichs ist jedenfalls die teuerste „Versorgungs“variante.

Ich wünsche Ihnen eine anregende Lektüre. 